\title{
Carotenoids: Importance in Daily Life_-Insight Gained from EPR and ENDOR
}

\author{
A. Ligia Focsan ${ }^{1}$ (D) - Nikolay E. Polyakov ${ }^{2,3} \cdot$ Lowell D. Kispert ${ }^{4}$
}

Received: 3 August 2020 / Revised: 31 December 2020 / Accepted: 14 January 2021 /

Published online: 20 March 2021

(c) The Author(s), under exclusive licence to Springer-Verlag GmbH, AT part of Springer Nature 2021

\begin{abstract}
Carotenoids are indispensable molecules for life. They are present everywhere in plants, algae, bacteria whom they protect against free radicals and oxidative stress. Through the consumption of fruits and vegetables and some carotenoid-containing fish, they are introduced into the human body and, similarly, protect it. There are numerous health benefits associated with the consumption of carotenoids. Carotenoids are antioxidants but at the same time they are prone to oxidation themselves. Electron loss from the carotenoid forms a radical cation. Furthermore, proton loss from a radical cation forms a neutral radical. In this mini-review, we discuss carotenoid radicals studied in our groups by various physicochemical methods, namely the radical cations formed by electron transfer and neutral radicals formed by proton loss from the radical cations. They contain many similar hyperfine couplings due to interactions between the electron spin and numerous protons in the carotenoid. Different EPR and ENDOR methods in combination with DFT calculations have been used to distinguish the two independent carotenoid radical species. DFT predicted larger coupling constants for the neutral radical compared to the radical cation. Previously, INDO calculations miss assigned the large couplings to the radical cation. EPR and ENDOR have aided in elucidating the physisorb, electron and proton transfer processes that occur when carotenoids are adsorbed on solid artificial matrices, and predicted similar reactions in aqueous solution or in plants. After years of study of the physicochemical properties of carotenoid radicals, the different published results start to merge together for a better understanding of carotenoid radical species and their implication in biological systems. Up until 2008, the radical chemistry in artificial systems was elucidated but the correlation between quenching ability and neutral radical formation was an inspiration to look for these radical species in vivo. In addition, the EPR spin-trapping technique has been applied to study inclusion complexes of carotenoids with different delivery systems.
\end{abstract}

A. Ligia Focsan

alfocsan@valdosta.edu

Extended author information available on the last page of the article 


\section{Carotenoids: Importance in Daily Life}

Carotenoids are long stick-like molecules synthesized biochemically from eight isoprene units (see Fig. 1). This highly conjugated system consisting of 9-11 double bonds and terminating in rings is responsible for the ability to transfer electrons throughout the molecule, acting like strings. This double bond conjugated system also determines which wavelengths of light will be absorbed and thus what color we observe for carotenoids-containing plants, vegetables, bacteria, or fungi. Carotenoids are pigments and give the red, orange or yellow color to these organisms. $\beta$-Carotene, for example, is the yellow or orange pigment abundant in carrots, sweet potatoes, butternut squash, pumpkin, apricots, peaches, cantaloupe and also present in dark green vegetables like spinach, kale, lettuce or broccoli. Among the carotenes, which are the hydrocarbon carotenoids, also called non-oxygenated carotenoids, $\beta$-carotene is distinguished by having beta-rings at both ends of the molecule (see Fig. 1). Similar to other carotenoids which are all known to have powerful antioxidant activities, $\beta$-carotene provides numerous health benefits including promoting skin health, lung health, eye health and even preventing cancer [1-3]. The difference between natural and synthetic $\beta$-carotene, for example, $\beta$-carotene from foods versus<smiles>CC1=C(/C=C/C(C)=C/C=C/C(C)=C/C=C/C=C(C)/C=C/C=C(C)/C=C/C2=C(C)CCCC2(C)C)C(C)(C)CCC1</smiles><smiles>CC1=C(/C=C/C(C)=C/C=C/C(C)=C/C=C/C=C(C)/C=C/C=C(C)/C=C/C2=C(C)CC(O)CC2(C)C)C(C)(C)CC(O)C1</smiles><smiles>CC1=CC(O)CC(C)(C)C1/C=C/C(C)=C/C=C/C(C)=C/C=C/C=C(C)/C=C/C=C(C)/C=C/C1=C(C)CC(O)CC1(C)C</smiles><smiles>CC(/C=C/C=C/C1(O)C(C)(C)CC(O)C(=O)C1(C)C)=C\C=C\C=C(C)\C=C\C=C(C)\C=C\C12OC1(C)C(=O)C(O)CC2(C)C</smiles><smiles>CC1=C(/C=C/C(C)=C/C=C/C(C)=C/C=C/C=C(C)/C=C/C=C(C)/C=C/C2=C(C)C(=O)C(O)CC2(C)C)C(C)(C)CC(O)C1=O</smiles>

Fig. 1 Carotenoid structures 
pharmacological supplements is still to be determined. However, foods containing carotenoids are associated with a reduced risk of lung, head and neck cancer [4, 5].

Zeaxanthin has also a symmetric structure that is essentially similar to that of $\beta$-carotene, except that it contains two extra hydroxyl groups, one on each of the two cyclohexene rings (see Fig. 1). Adding oxygen atoms into the structure makes zeaxanthin a xanthophyll, which are oxygen derivatives of carotenes. Zeaxanthin is also present in fruits and dark green vegetables but it is also known to be the macular pigment in human eyes where it protects the eye against excessive light. Similarly, it protects plants against photoxidative damage. Together with violaxanthin it is part of the xanthophyll cycle which protects plants against photoxidative damage. Structurally violaxanthin contains epoxide groups (see Fig. 1). During light stress, violaxanthin is enzymatically converted to zeaxanthin, which plays a direct photoprotective role. We have observed that the photoprotective role of zeaxanthin is in agreement with the formation of neutral radicals from the radical cation, radical species that could act as an additional quencher to the known quenching function of the radical cation. Violaxanthin does not form neutral radicals and is not known to be a photoprotective agent.

Lutein, which is just an isomer of zeaxanthin, with a double bond moved one position and is also known as a photoprotective agent. It is also a predominant carotenoid in the macular pigment, along with zeaxanthin, and meso-zeaxanthin. Lutein supplementation ( 10 or $20 \mathrm{mg} /$ day) for more than 6 months can significantly improve macular pigment optical density and visual acuity in patients with agerelated macular degeneration [6].

Astaxanthin, another xanthophyll containing carbonyl groups besides the hydroxyl groups is the keto-carotenoid (see Fig. 1) present in certain algae-like Haematococcus pluvialis. During stress like high light exposure, absence of nutrients or in the presence of salt, the algae remarkably produces astaxanthin to protect itself. Astaxanthin is also the carotenoid that gives the pink or red color to aquatic animals like shrimp, lobster, salmon, fish eggs, and trout. This pigment is also responsible for the beautiful pink color in flamingo. Astaxanthin is known as a super-antioxidant able to repair cellular damage caused by free radicals. Its consumption can result in various health benefits and has great application potentials in cosmetics, nutritional health products, as well as medicines. The amount of natural astaxanthin is limited and delivery systems such as emulsions, liposomes and nanoparticles are developed to improve the bioavailability and stability of astaxanthin [7, 8].

\section{Carotenoid Radicals and Their Role in Nature}

Carotenoids have a large extinction coefficient about $10^{5} \mathrm{M}^{-1} \mathrm{~cm}^{-1}$ and only small amounts $(\mu \mathrm{g})$ are sufficient for absorption measurements, for example. Similar amounts are sufficient for electrochemical measurements such as cyclic voltammetry. A millimolar carotenoid concentration in methylene chloride would generate a cyclic voltammogram indicating peaks for oxidation $(1,2)$ and reduction $(3,4,5)$ of the carotenoid like in Fig. 2. There are a multitude of reactions like heterogeneous 

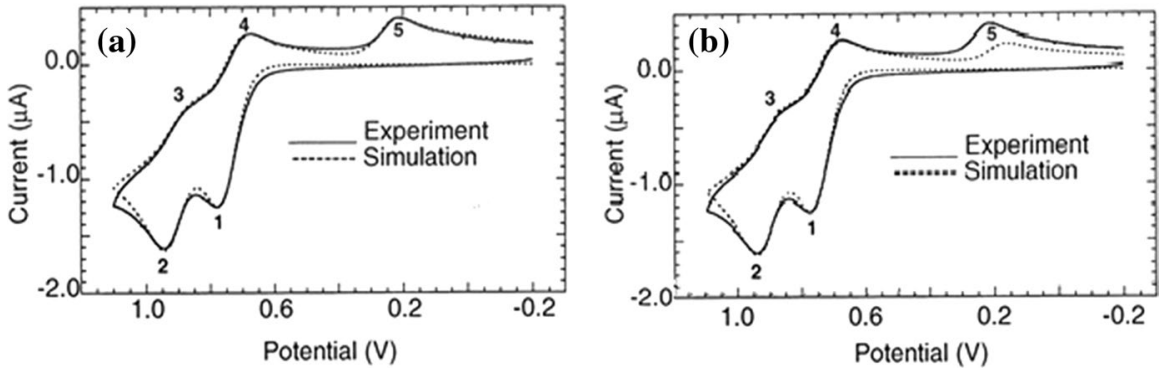

Fig. $2 \mathrm{CV}$ of carotenoid: a considering the deprotonation of the radical cation to form neutral radicals at peak 5 and $\mathbf{b}$ without considering deprotonation of the radical cation. Adapted from: [13]. Peak 5 demonstrates the formation of neutral radicals Car' in solution

\begin{tabular}{|c|c|c|}
\hline $\begin{array}{l}\text { Heterogeneous electrode reactions: } \\
\text { (1) Car } \stackrel{E_{1}^{0_{1}}}{\rightleftharpoons} \mathrm{Car}^{++}+\mathrm{e}^{-} \\
\text {(2) } \mathrm{Car}^{++} \stackrel{E_{2}^{0_{2}}}{\rightleftharpoons} \mathrm{Car}^{2+}+\mathrm{e}^{-} \\
\text {(3) } \mathrm{Car}^{+}+\mathrm{e}^{-} \stackrel{E^{0_{3}}}{\rightleftharpoons} \mathrm{Car}^{-}\end{array}$ & $\begin{array}{l}\text { Homogeneous reactions: } \\
\begin{array}{l}\text { (4) } \mathrm{Car}^{2+}+\mathrm{Car} \stackrel{K_{\mathrm{com}}}{\rightleftharpoons} 2 \mathrm{Car}^{++} \\
\text {(5) } \mathrm{Car}^{2+} \stackrel{K_{\mathrm{do}}}{\rightleftharpoons} \mathrm{Car}^{+}+\mathrm{H}^{+} \\
\text {(6) } \mathrm{Car}^{++} \stackrel{K_{\mathrm{dp}}}{\rightleftharpoons} \mathrm{Car}^{\circ}+\mathrm{H}^{+}\end{array}\end{array}$ & $\begin{array}{l}\text { Side reactions: } \\
\begin{aligned} \text { (7) Car }{ }^{+} \rightarrow \text { Unknown Products } \\
\text { (8) } \mathrm{Car}^{2+} \rightarrow \text { Unknown Products } \\
\text { (9) } \mathrm{Car}^{+} \rightarrow \text { Unknown Products } \\
\text { (10) Car } \rightarrow \text { Unknown Products }\end{aligned}\end{array}$ \\
\hline
\end{tabular}

Scheme 1 Electrochemical reactions of carotenoids. Adapted from [13]

electrode reactions, homogeneous solution reactions and other side reactions taking place in solution. To simulate the $\mathrm{CV}$ of a carotenoid about 10 reactions and numerous parameters $\left(E_{1,}^{0} E_{2,}^{0} E_{3}^{0}, K_{\mathrm{com}}, K_{\mathrm{dp}}, K_{\mathrm{dp}}^{\prime}, k_{\mathrm{f}}, D_{1}\right)$ need to be considered (see Scheme 1). Peaks 1 and 2 are due to oxidation of carotenoid molecule (Car) to the radical cation $\left(\mathrm{Car}^{-+}\right)$and oxidation of the radical cation to a dication $\left(\mathrm{Car}^{2+}\right)$, associated with forward reactions 1 and 2 in Scheme 1. Peaks 3 and 4 are due to the reduction of the dication and radical cation, associated with backward reactions 2 and 1, respectively. Peak 5, as deduced from the simulation is the effect of deprotonation of both radical cation (weak acid, $\mathrm{p} K_{\mathrm{a}}$ approx. 4-7) and dication (strong acid, $\mathrm{p} K_{\mathrm{a}}$ approx. -2 ) to build up neutral radical species (Car). Figure 2 shows that to fit peak 5 contribution of deprotonation from the radical cation to form neutral radicals is needed. A recent review [9] gathers the first oxidation potentials of different carotenoids determined in Prof. Kispert's lab and calibrated against ferrocene (details are given in Refs. $[10,11]$ ), and discusses the increased antioxidant activity with increasing the oxidation potential favored by a nonpolar environment and disfavored by $\mathrm{H}$-bonding of the carotenoid within a host [12].

Peak 5 in the $\mathrm{CV}$ in Fig. 2 demonstrates that deprotonation of the radical cation occurs in solution. As confirmed by DFT calculations, proton loss in carotenoids is most favorable at the ends of the radical cation [14]. This feature was especially useful in analyzing crystal structures of light-harvesting complexes (LHCs) for possible proton loss from a radical cation of carotenoids present. Figure 3 shows the crystal structure of the major LHCII with four carotenoids. For proton loss to occur in vivo the orientation of the carotenoids in thylakoid membrane is especially important. Figure $3 \mathrm{a}$ shows the orientation of the carotenoids in LHCII and the positions for 

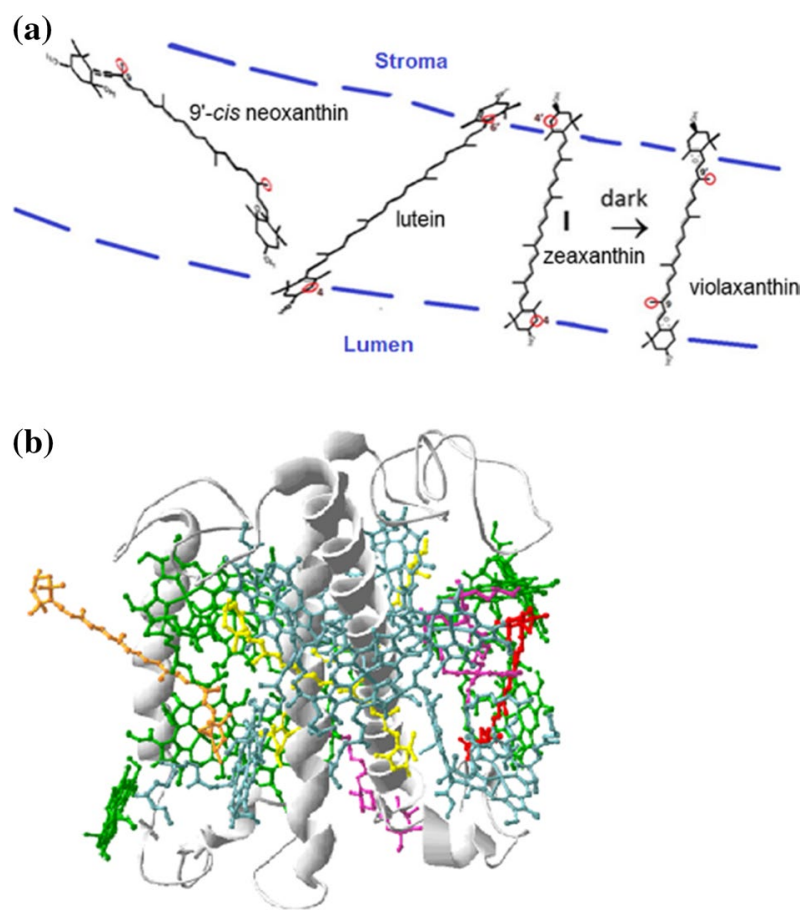

Fig. 3 a Schematic of the position of carotenoids in LHCII. b Crystal structure of LHCII. Adapted from [17]

proton loss (represented by red circles) occurring at the ends of the radical cations of these carotenoids. Proton loss would be facilitated by proton acceptors in nearby thylakoid lumen and stroma. Figure $3 \mathrm{~b}$ shows the LHCII crystal structure with carotenoids in the same positions as in Fig. 3a. Zeaxanthin which is accumulated under sunlight and represented in red in Fig. 3b, converts to violaxanthin under low light/ dark. Two lutein molecules are found in a cross position in yellow, and 9'-cis neoxanthin is depicted in orange. For zeaxanthin and lutein, which are known as quenchers, proton loss is possible at both ends of the radical cations. $9^{\prime}$-cis neoxanthin is buried in the nonpolar environment and its epoxy on one end and the allene bond would prevent proton loss at the C5 and C5'-methyl groups on the ends by reducing the conjugation so crucial for the neutral radical stability [14]. Violaxanthin also contains epoxy groups at both ends which would also prevent proton loss [15]. It was noted that the ability to form neutral radicals by deprotonation of the radical cation is correlated with the quenching/nonquenching properties of the four carotenoids present in this structure. More exactly, zeaxanthin and lutein that are known to be quenchers from the neutral radicals by proton loss from the radical cation, while violaxanthin and 9'-cis neoxanthin do not [14-16].

We have concluded that zeaxanthin and lutein radical cations' ability to deprotonate and form neutral radicals could be linked to their quenching activity. It was thus proposed an additional quenching mechanism [15] involving neutral radicals 
of zeaxanthin and lutein. For zeaxanthin, for example, a charge transfer complex $\left(\mathrm{Zea}^{+}{ }^{+} . \mathrm{Chl}^{-}\right)$forms in presence of intense sunlight present on a clear bright sunny day. Deprotonation of the radical cation to the neutral radical, Zea ${ }^{+} \leftrightarrow \mathrm{Zea}^{-}+\mathrm{H}^{+}$, easily happens because the $\mathrm{p} K_{\mathrm{a}}$ of $\mathrm{Zea}^{++}$is 4 which favors proton loss as a weak acid. Reprotonation of Zea is electrochemically [13] unfavorable, so a lifetime of the neutral radical could increase to ms or longer and exists after collapse of $\mathrm{Zea}^{+}{ }^{+} . . \mathrm{Chl}^{-}$ complex. The neutral radical Zea formed is able to quench excited $\mathrm{Chl}^{--}$. The lifetime of $\mathrm{Zea}^{+}{ } . . \mathrm{Chl}^{-}$was measured to be about $150 \mathrm{ps}$. Chl ${ }^{-}$charge migration [18] causes the delay in the collapse of $\mathrm{Zea}^{+}{ }^{+} . . \mathrm{Chl}$, allowing a small amount of $\mathrm{Zea}^{+}$to deprotonate and ready to quench other excited $\mathrm{Chl}^{*}$ formed upon continuous light irradiation [19]. The xanthophyll cycle operates in lab light or a cloudy day; violaxanthin which has ends protected by epoxide groups preventing proton loss, converts to Zea, for Zea ${ }^{+}$and neutral radical Zea to be formed if there is too much light. In the dark, zeaxanthin converts to violaxanthin during xanthophyll cycle preventing proton loss but violaxanthin is ready to absorb light and photosynthesis continues over a wide range of light intensity. An organized assembly favors Zea which is absolutely necessary for the formation of the neutral radicals. Betacarotene, for example, would not be adequate for the organized assembly nor would the cis isomer of Zea.

A neutral radical like Zea would be able to quench the excited state from a neighboring chlorophyll. Quenching by free radicals is important in liquids and solids and forms the basis for fluorescence detection of reactive oxygen species. Quenching of fluorescence by $\mathbf{J}$ exchange for either an excited single or triplet state has been accomplished by attaching a stable nitroxide neutral radical as far away as $9 \AA$ from a fluorescing molecule [20]. In Fig. 4 is shown the crystal structure of the minor light-harvesting complex CP29 (Protein Data Bank 3PL9) where the xanthophyll is located between adjacent chlorophylls. One end is next to a chlorophyll permitting a charge transfer complex to be formed upon light exposure [21]. The negative charge of chlorophyll migrates over adjacent chlorophyll [18] over the 150 ps time during which time the radical cation formed can transfer a proton to the water molecule located within $9 \AA$ thus forming a quenching neutral radical. This radical species
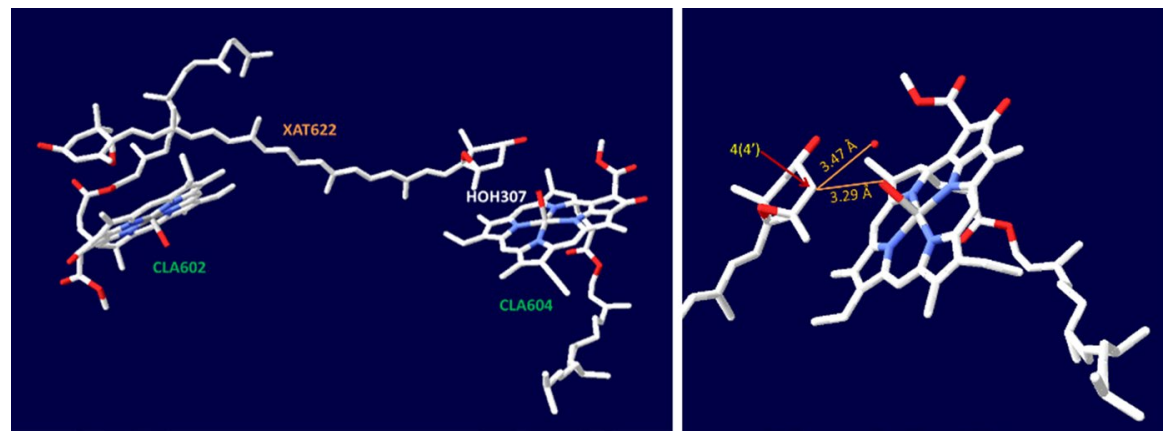

Fig. 4 Xanthophyll in close proximity (less than $9 \AA$ ) of a water molecule. From the structure of minor light-harvesting complex CP29 (Protein Data Bank 3PL9) 
quenches excited chlorophyll during 150 ps lifetime of the charge-transfer complex. The crystal structure of CP29 (Protein Data Bank 3PL9) also shows a lutein molecule with $\mathrm{Chl}$ molecules and water molecules situated in close proximity (less than $9 \AA$ ) of both ends of the carotenoid.

Zeaxanthin which accumulates in the presence of light is a very special and necessary carotenoid. It has a low oxidation potential and thus it can be easily oxidized to form $\mathrm{Zea}^{++}$. A molecule with a higher oxidation potential like astaxanthin [11] for example, would not form a radical cation as easily and would favor scavenging of radicals like $\mathrm{OH}, \mathrm{OOH}$ instead [22]. $\mathrm{Zea}^{+}$is a weak acid $\left(\mathrm{p} K_{\mathrm{a}} \sim 4\right)$ and deprotonation is favored as an equilibrium. Its deprotonation occurs at the 4-C or 4'-C end terminal to form the neutral radical. Zea neutral radical is long lived and reprotonation is difficult, given the unfavorable potentials. The terminal $\mathrm{OH}$ groups facilitate hydrogen bonding with water. In contrast, a molecule such as $\beta$-carotene would not work in the presence of proton acceptors. Terminal $\mathrm{OH}$ groups also facilitates $\mathrm{H}$-aggregation in solvents containing water causing a $100 \mathrm{~nm}$ blue shift [23]. This aggregation reduces reaction with free radicals like $\mathrm{OH}, \mathrm{OOH}$ and makes zeaxanthin prone to oxidation by $\mathrm{Fe}^{3+}$ or other oxidants to form zeaxanthin radical cation, $\mathrm{Zea}^{+}$. The oxidation potential of zeaxanthin was determined to be $571 \pm 11 \mathrm{mV}$, essentially identical to that of $\beta$-carotene $567 \pm 4 \mathrm{mV}$ by Niedzwiedzki et al. [24]. We have also found a value for the oxidation potential of $\beta$-carotene $634 \pm 4 \mathrm{mV}$ [10] when compared to other carotenoids which explained its prooxidant effect and forming the radical cation in reaction with $\mathrm{Fe}^{3+}$ [25]. The oxidation potential of violaxanthin $681 \pm 14 \mathrm{mV}$ was found to be higher than that of zeaxanthin implying that the natural selection of zeaxanthin for its photoprotective role over violaxanthin is owed, at least in part, to its ability to be easier oxidized forming Zea ${ }^{+}$. A study of Arabidopsis thaliana as a function of varying light intensity (bright sunny day, cloudy day, lab light, dark drawer) has demonstrated that zeaxanthin neutral radical can form in an organized assembly when $\mathrm{Zea}^{+}{ }^{+} . . \mathrm{Chl}^{-}$was generated in bright sun light [21]. Our review article [19] summarizes all the key concepts learned about zeaxanthin radical cations and neutral radicals that are necessary for photoprotection. All E zeaxanthin isomer is the only carotenoid that will work best. As mentioned above, an organized assembly can trigger deprotonation of its weak acid radical cation at the terminal 4-C positions giving rise to a long lifetime of neutral radical because reprotonation is unfavorable. Little protonation, fragmentation or epoxidation as observed for the Z-isomers [21].

\section{EPR and ENDOR Studies of Carotenoid Radicals Correlated with DFT Studies}

Carotenoids have a long conjugated chain in which $\pi$ electrons are delocalized. Thus carotenoid radicals contain a large number of anisotropic $\alpha$-protons and isotropic $\beta$-protons from rotating methyl groups. Radicals formed, namely the radical cations formed by electron transfer and the neutral radicals formed by proton loss from the radical cations, contain lots of similar couplings so we used different electron paramagnetic resonance (EPR) and electron-nuclear double resonance (ENDOR) 
techniques to distinguish and identify them. It is known from electrochemical studies in the solution discussed above that radical cations and dications are formed upon oxidation, and the radical cations can lose protons in water to form neutral radicals. Also, the dication loses a proton and upon reduction forms the neutral radical. Similar reactions occur in powders when carotenoids are adsorbed on silica alumina or inserted into MCM-41 containing silanol groups.

Carotenoids (Car) exhibit oxidation potentials measured in our lab between 0.63 and $0.94 \mathrm{~V}$ vs SCE [12]. Their oxidation potential varies depending on the substituents, increasing with the presence of electron-accepting groups [9]. As antioxidants, carotenoids are a source of donating electrons to a physisorb catalyst solid surface like alumina, silica-alumina or MCM-41. Silanol groups are electron acceptors of carotenoids and by adsorbing carotenoids on such surfaces, EPR and ENDOR techniques gave insight into the radical chemistry that occurs on catalyst surfaces. When an electron is transferred to the catalyst, a radical cation is formed. Radical cations of carotenoids are weak acids, exhibiting $\mathrm{p} K_{\mathrm{a}}$ between 4 and 9 . With increasing the oxidation potential of the carotenoid the $\mathrm{p} K_{\mathrm{a}}$ increases, or proton donor ability decreases. A carotenoid with lower oxidation potential like $\beta$-carotene would form a radical cation easier and would have a higher proton donor ability. Initially, the carotenoid molecule donates an electron to the silica alumina to form the radical cation. The electron can be transferred to the Lewis acidic site (LAS), while the $\mathrm{Al}$ in the lattice generates Bronsted acidic sites (BAS) which stabilizes the radical cation. The radical cation which is a weak acid can donate a proton to form a neutral radical, this being enhanced under light irradiation.

The formation of carotenoid radical cations on activated alumina and silica alumina which includes electron transfer from adsorbed carotenoid molecules to the LAS was established using the EPR spin probe with TEMPONE and ENDOR techniques [26]. The role of the Al LAS in the stabilization of the formed radical cations was supported by the correlation between the LAS content on the activated surfaces and the maximum concentration of the generated radical cations. ENDOR is most simple defined as EPR-detected NMR where the EPR signal is measured at a fixed magnetic field and its intensity is varied by the applied scanned radio frequency, and makes use of the hyperfine couplings between unpaired electrons and the neighboring nuclei. The ENDOR peak at ca. $6.5 \mathrm{MHz}$ obtained for both canthaxanthin adsorbed on alumina and silica-alumina, and a mixture of canthaxanthin $\mathrm{AlCl}_{3}$ solution, was attributed to $\mathrm{Al}$ nuclear frequency at $3.65 \mathrm{MHz}$ plus hyperfine coupling of $7 \mathrm{MHz}$. Detection of a hyperfine doublet for matrix ENDOR, instead of a single line at the $3.65 \mathrm{MHz}$ Larmor frequency for $\mathrm{Al}$ indicated the formation of strong complexes between carotenoid molecules and Al LAS on the surface.

The $9 \mathrm{GHz}$ EPR spectrum of a carotenoid radical is not resolved due to the numerous EPR lines for the different protons (a carotenoid contains approx. 50 $\mathrm{H}$ atoms). The interaction of an electron with a proton gives a coupling of about $1400 \mathrm{MHz}$ and for a $\mathrm{CH}$ fragment that carotenoids contain, the unpaired spin distribution is reduced to $4 \%$ to about $56 \mathrm{MHz}$. For approximately 10 of these $\mathrm{CH}$ fragments in a carotenoid, the distribution of the unpaired electron over the $\pi$ system would give couplings around 5.6 MHz. There is symmetry for some carotenoids and the rotation of methyl groups reduces the number of EPR lines but still the structure 
is not resolved at $9 \mathrm{GHz}$ due to the numerous EPR lines for the different protons. At higher mw frequency $(327 \mathrm{GHz})$ the structure of a carotenoid starts to resolve indicating the cylindrical symmetry of a $\pi$ radical.

Because there was a need to distinguish the spectrum of the radical cation from that of the neutral radicals, the approach was to form stable radicals for hours on catalysts like silica-alumina or MCM-41, and study them using different ENDOR procedures. Continuous wave $(\mathrm{CW})$ ENDOR of carotenoids adsorbed on silica alumina measured at $120 \mathrm{~K}$ was performed, as the methyl groups rotate very rapidly giving the isotropic hyperfine couplings, and in fact, the powder spectrum was resolved. To determine the precise values of the hyperfine interactions, the analysis of ENDOR spectra required density functional theory (DFT) simulations. It was determined using calculated DFT hyperfine couplings that a mixture of radical cations $\mathrm{Car}^{+}{ }^{+}$and neutral radicals Car' was formed for all carotenoids studied. Any line above $19 \mathrm{MHz}$ was assigned to the neutral radicals, below that value peaks indicated a mixture of radical cations and neutral radicals. For example, the simulated and experimental spectrum for an irradiated sample of betacarotene on silica alumina showed contribution from both radical species [27].

In a non-irradiated sample (see Fig. 5) the lines were assigned using DFT-calculated couplings to the radical cation $\mathrm{Car}^{+}$only. There are no lines above $19 \mathrm{MHz}$ because the radical cation is stabilized by BAS. The typical isotropic hyperfine coupling for a carotenoid radical cation is around $8 \mathrm{MHz}$. However, light irradiation of the samples and the presence of metals in the matrix produced more neutral radicals, with larger values of the isotropic hyperfine couplings. Figure 6 shows CW ENDOR powder spectrum of canthaxanthin in Al-MCM41 and in Fe-MCM41 irradiated at $77 \mathrm{~K}$ and measured at $120 \mathrm{~K}$. Couplings of $13 \mathrm{MHz}$ identified the carotenoid neutral radicals Car of canthaxanthin as deduced from the ENDOR lines at approx. $22 \mathrm{MHz}$. Also note that the concentration of the neutral radicals Car' depends on the metal: it is higher in the presence of $\mathrm{Al}$ than in the presence of $\mathrm{Fe}$.

Besides the CW ENDOR experiments that use a continuous microwave source, we performed pulsed ENDOR experiments which use a microwave pulse sequence predominately developed by Mims and Davies. The experimental Davies ENDOR of
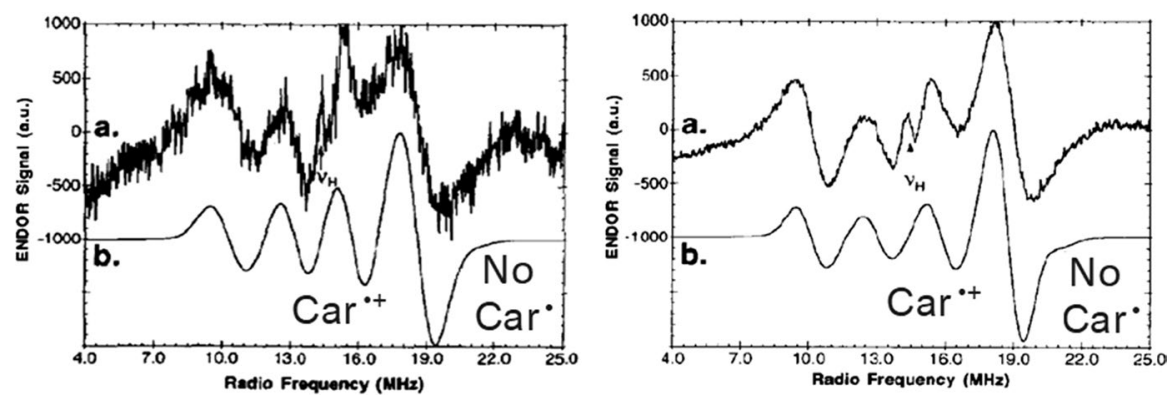

Fig. $5 \mathrm{CW}$ ENDOR powder spectrum of $\beta$-carotene (left) and canthaxanthin (right) adsorbed on activated silica alumina solid support at $120 \mathrm{~K}$ in the absence of UV light a experimental and $\mathbf{b}$ simulated $\mathrm{Car}^{+}$. Adapted from Ref. [28] 

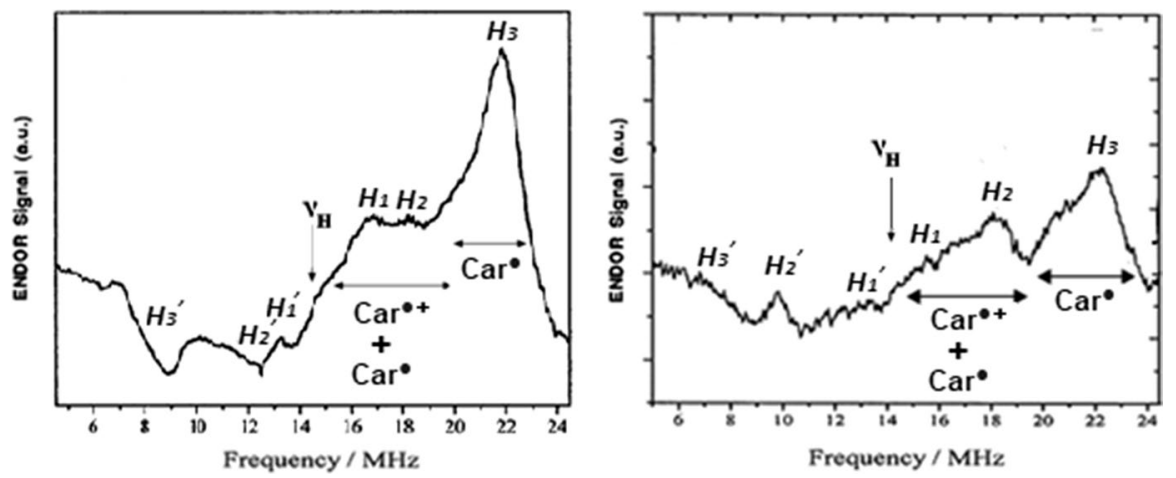

Fig. 6 CW ENDOR powder spectrum of canthaxanthin in Al-MCM41 (left) and in Fe-MCM-41 (right) irradiated at $77 \mathrm{~K}$ and measured at $120 \mathrm{~K}$ (adapted from Refs. [29, 30], respectively)

carotenoids run at $20 \mathrm{~K}$ consists of two broad regions, each composed of many overlapping lines spaced around the free proton frequency [14, 15]. It is not possible to deconvolute the poorly resolved spectrum as shown in Fig. 7a in red for the zeaxanthin radicals on silica-alumina. The spectra in Fig. 7b-d are simulations using DFT generated hyperfine couplings for radicals optimized with B3LYP/6-31G** and calculated with B3LYP/Ahlrichs. Figure 7d indicates the simulation of just the radical cation including both isotropic and anisotropic hyperfine couplings. Figure $7 \mathrm{c}$ shows the peaks A through $\mathrm{H}$ for both the radical cation and all neutral radicals considering just the isotropic hyperfine couplings due to the $\beta$-methyl protons in methyl groups. These peaks start to broaden when the anisotropic components due to $\alpha$-protons are added to the isotropic components in Fig. 7b, to better match the experimental spectrum. In conclusion, to match the experimental Davies ENDOR spectrum, the anisotropic hyperfine

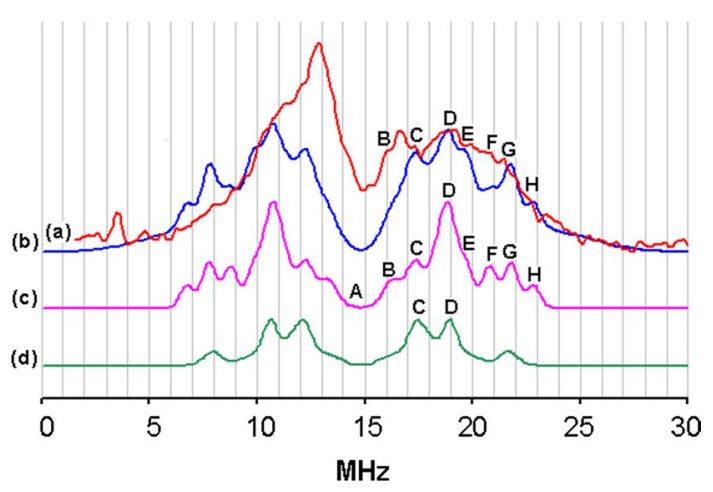

Fig. 7 Davies ENDOR spectra of zeaxanthin radicals on silica-alumina. Taken from [15]. (a) Experimental and (b)-(d) simulations using DFT generated couplings (B3LYP/Ahlrichs // B3LYP/6-31G**). (b) uses both isotropic $\beta$-methyl proton and anisotropic $\alpha$-proton couplings for all radicals (radical cation and neutral radicals); (c) uses only isotropic $\beta$-methyl proton couplings for all radicals (radical cation and neutral radicals); (d) uses isotropic $\beta$-methyl proton and anisotropic $\alpha$-proton couplings for the radical cation only 
couplings needed to be added to the isotropic couplings for the radical cation and for the neutral radicals, proving that a mixture of radicals was formed.

Davies spectra of carotenoids indicated that a mixture of radical cations and neutral radicals was formed but due to the poor resolution we turned to Mims ENDOR as a complementary method to study carotenoids [14, 31, 32]. Using this method, by varying the delay time $(\tau)$ which is the time between first and second pulse in the Mims ENDOR experiment, features in the spectrum can be deleted where the ENDOR amplitude goes to zero causing a blind spot. The ENDOR amplitude goes to zero according to $1-\cos (2 \pi A \tau)$ where $A$ is the hyperfine coupling, so having DFT calculated hyperfine couplings and selecting different delay times can erase or show the presence of certain peaks. This was instrumental in demonstrating that peaks corresponding to the carotenoid neutral radicals appear at the edges of the spectrum at high- and low-frequency. In other words, by varying the delay time we could map out the neutral radicals differentiating them from the radical cation [14]. The examined properties by Davies and Mims ENDOR sustained by simulations using DFT calculated hyperfine couplings, along with the known crystal structure of the LHC II suggest the absence of the neutral radicals of violaxanthin [15] and 9'-cis neoxanthin [14] available for quenching the excited states of $\mathrm{Chl}$, consistent with their observed nonquenching properties. This was possible by identifying isotropic coupling constants from DFT calculations for the radical cation $\mathrm{Car}^{+}$and for the neutral radicals Car. As a consequence of deprotonation of the radical cation, the unpaired electron spin distribution changes so that larger $\beta$-methyl proton couplings occur for the neutral radicals $(13-16 \mathrm{MHz})$ than for the radical cation (7-10 MHz), providing a means to differentiate between these carotenoid radicals [31].

\section{EPR Spin-Trapping Study of the Antioxidant and Pro-oxidant Activities of Carotenoids}

Carotenoids are natural antioxidants which protect human body from diseases caused by toxic free radicals. The scavenging ability of carotenoids towards free peroxyl radicals $\mathrm{OOH}$ was investigated in Refs. [23, 26] using the well-known Fenton reaction [33] in the presence of DMSO and spin trap PBN (see Scheme 2). At low $\mathrm{H}_{2} \mathrm{O}_{2}$ concentration only spin adduct $\mathrm{PBN}-\mathrm{CH}_{3}$ was detected with EPR parameters $a(\mathrm{H})=3.4 \mathrm{G}$ and $a(\mathrm{~N})=14.9 \mathrm{G}$. At higher $\mathrm{H}_{2} \mathrm{O}_{2}$ concentration $(0.5 \mathrm{M})$ the reaction of $\mathrm{CH}_{3}$ radicals with $\mathrm{H}_{2} \mathrm{O}_{2}$ results in the disappearance of the $\mathrm{PBN}-\mathrm{CH}_{3}$ adduct, and appearance of another adduct which was assigned to the PBN-OOH spin adduct $[a(\mathrm{H})=2.3 \mathrm{G}$ and $a(\mathrm{~N})=13.9 \mathrm{G}]$ [22]. The additional confirmation of the PBN-OOH adduct formation was obtained using the superoxide dismutase test.

Scheme 2 Fenton reaction in the presence of DMSO

$$
\begin{aligned}
& \mathrm{Fe}^{2+}+\mathrm{H}_{2} \mathrm{O}_{2} \longrightarrow \mathrm{Fe}^{3+}+{ }^{\circ} \mathrm{OH}+\mathrm{OH}^{-} \\
& \cdot \mathrm{OH}+\mathrm{DMSO} \longrightarrow \mathrm{CH}_{3}+\mathrm{CH}_{3}(\mathrm{OH}) \mathrm{SO} \\
& { }^{\cdot} \mathrm{CH}_{3}+\mathrm{H}_{2} \mathrm{O}_{2} \longrightarrow{ }^{\circ} \mathrm{OOH}+\mathrm{CH}_{4}
\end{aligned}
$$


The scavenging ability of carotenoids was measured as a relative scavenging rate by carotenoid (Car) and spin trap (ST). These values were determined from concentration dependence of spin adduct yield $(A)$ by using the equation:

$$
\frac{A_{0}}{A}=\frac{k_{\mathrm{ST}}[\mathrm{ST}]+k_{\mathrm{Car}}[\mathrm{Car}]}{k_{\mathrm{ST}}[\mathrm{ST}]},
$$

where $A_{0}$ is spin adduct yield at zero carotenoid concentration, and $k_{\mathrm{Car}}$ and $k_{\mathrm{ST}}$ are the reaction rate constants of carotenoid and spin trap with a free radical. It was observed, that $k_{\mathrm{Car}}$ values depend strongly on the redox properties of carotenoid and increase with increasing of their oxidation potentials [22]. According to our results, $\beta$-carotene shows the worst antioxidant ability among the carotenoids under study. The values of $k_{\mathrm{Car}} / k_{\mathrm{ST}}$ change from 0.6 for $\beta$-carotene and zeaxanthin to 24 for 7 -apo-7,7-dicyano- $\beta$-carotene (see Table 1 ). Figure 8 demonstrates the decrease of spin adduct yield with increase the carotenoid concentration as the result of scavenging process.

The presence of carotenoid in the Fenton reaction system can results, however, not only in a decrease of the free radical concentration but the reduction of $\mathrm{Fe}^{3+}$ to $\mathrm{Fe}^{2+}$ by carotenoid according to $\mathrm{Car}+\mathrm{Fe}^{3+\rightarrow} \mathrm{Car}^{+}+\mathrm{Fe}^{2+}$ reaction.

The corresponding radical cation $\mathrm{Car}^{+}$was detected as a product of this reaction. In the presence of excess of $\mathrm{H}_{2} \mathrm{O}_{2}$ this reaction will result in repetition of the redox

Table 1 First oxidation potentials for selected carotenoids and the relative scavenging ability towards peroxyl radicals [9-11, 34, 35]
Fig. 8 EPR spectra of PBN$\mathrm{OOH}$ spin adduct in the presence of $8^{\prime}$-apo- $\beta$-caroten8 -oic acid. Concentration of $\mathrm{PBN}=10 \mathrm{mM} ; \mathrm{Fe}^{2+}=1 \mathrm{mM}$; $\mathrm{H}_{2} \mathrm{O}_{2}=500 \mathrm{mM}$ in DMSO (from Ref. [36])

\begin{tabular}{lll}
\hline Carotenoid & $E^{0}{ }_{1}$ vs. SCE & $k_{\text {car }} / k_{\text {ST }}$ \\
\hline$\beta$-Carotene & $0.634 \pm 0.001$ & 0.64 \\
Canthaxanthin & $0.775 \pm 0.001$ & 1.96 \\
Astaxanthin & $0.768 \pm 0.001$ & 2.6 \\
Astaxanthin monoester & $0.774 \pm 0.001$ & 2.5 \\
8'-Apo- $\beta$-carotene-8'-al & $0.814 \pm 0.005$ & 3.22 \\
Ethyl-8'-apo- $\beta$-caroten-8'-oate & $0.816 \pm 0.005$ & 12.20 \\
7'-Apo-7',7'-dicyano- $\beta$-carotene & $0.833 \pm 0.005$ & 23.60 \\
\hline
\end{tabular}

The values $k_{\mathrm{Car}} / k_{\mathrm{ST}}$ were calculated from the linear dependence of the plot $\left(A_{0} / A-1\right)$ vs. [Car]

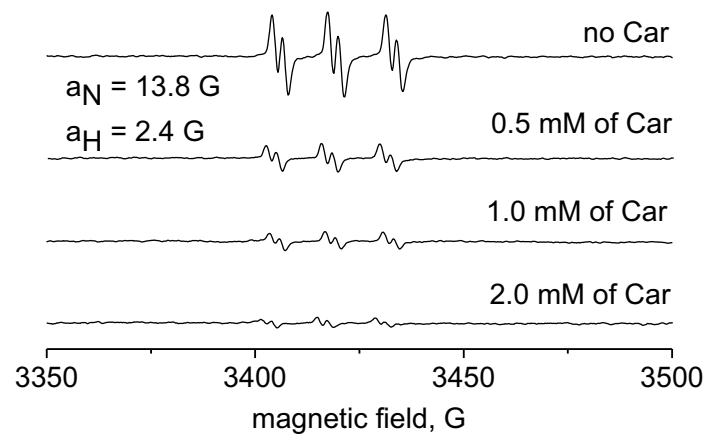


Scheme 3 Carotenoid driven

Fenton reaction

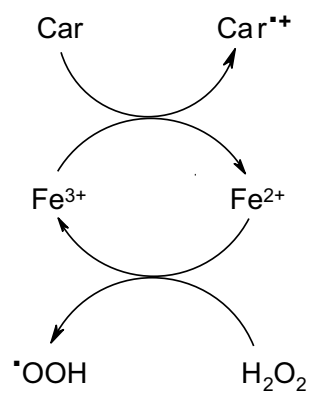

cycle of the Fenton process and production of an additional portion of free radicals (pro-oxidant effect, see Scheme 3) [25].

The appearance of this "carotenoid driven Fenton reaction" depends on the comparative rates of the carotenoid reactions with free radical and ferric ion [25]. From this point of view, the pro-oxidant effect of carotenoid should increase with decreasing in its oxidation potential. Beta-carotene has the lowest oxidation potential among carotenoids. In addition, the effectiveness of carotenoid reaction with free radicals will decrease with a decrease in the free radical concentration and the scavenging rate constant. We can assume that any additional effective decay channel for free radicals will enhance the pro-oxidant effect of carotenoids. In living cells, it might be the reaction with lipids or other biological targets. The role of Fenton-like processes in the generation of toxic free radicals is now being widely discussed $[37,38]$ and refs therein].

\section{Inclusion Complexes of Carotenoids with Watersoluble Oligosaccharides and Polysaccharides: The Way to Improve Carotenoid's Bioavailability, Stability and Antioxidant Activity}

Despite the importance of carotenoids in daily life, their practical application is restricted by extremely high sensitivity to environment factors such as temperature, sunlight, oxygen, and metal ions, as well as by extremely low water solubility. Nanoencapsulation of carotenoids into water soluble polymers, micelles or liposomes is an effective strategy to overcome these problems and to improve their stability under storage and processing. Numerous examples are described in several recent reviews [39-44]. Also, nanoencapsulation enhances bioavailability of carotenoids via modulating their release kinetics from the delivery system, influencing the solubility and absorption. In the present paper, we present the data obtained in Prof. Kispert's and Dr. Polyakov's labs on the reactivity of carotenoids encapsulated into various nanoparticles.

Due to their lipophilicity, carotenoids easily form supramolecular inclusion complexes of "host-guest" type with water-soluble nanoparticles. Such nanoparticles have hydrophilic surface and hydrophobic interior suitable for lipophilic "guest" molecules. It was demonstrated that incorporation of carotenoids into the "host" 
nanoparticles results in noncovalent binding between the carotenoid and "host" macromolecule, and can significantly change the physical and chemical properties of carotenoids [39, 42]. Most of our studies were performed with natural polysaccharide arabinogalactan (AG), oligosaccharides cyclodextrins (CD) and natural saponin glycyrrhizic acid (GA) [23, 36, 45-50]. We have applied EPR and optical absorption spectroscopy to investigate how complexation with AG, CD and GA can affect the aggregation ability of the xanthophyll carotenoids zeaxanthin, lutein, and astaxanthin, their photostability, and antioxidant activity.

Cyclodextrins are the cyclic oligosaccharides derived from starch and are the most developed delivery systems [51]. CDs have a hydrophilic shell and hydrophobic core suitable for the inclusion of lipophilic "guest" molecules of appropriate size. The most common CDs are $\alpha-C D, \beta-C D$ and $\gamma-C D$ which have a different number of glucopyranose units, 6, 7 and 8 units, and different ring diameters of 6,8 and $10 \AA$, respectively. Our NMR, EPR and optical studies showed that incorporation of carotenoids into $\mathrm{CD}$ cavity protects the carotenoid from reactive oxygen species, but decreases considerably the antioxidant ability of the carotenoid [36]. Moreover, carotenoid-CD complexes form water dispersions, rather than solutions. The reduced color intensity significantly decreases the use of carotenoid-CD complexes, at least as food colorants. This is why we used relatively water-soluble carotenoid, 8 '-apo- $\beta$-caroten- 8 '-oic acid, to estimate the antioxidant activity of carotenoid-CD complexes. In contrast with Fig. 9 no decrease in spin adduct yield was observed for CD complex of this carotenoid. Moreover, the appearance of pro-oxidant effect (increase of spin adduct yield) in the presence of carotenoid has been detected. As it was shown in Refs. [25, 36], the appearance of the pro-oxidant effect for cyclodextrin complexes of carotenoids can be attributed to chain elongation by the reaction of carotenoid with $\mathrm{Fe}^{3+}$ ions.

Arabinogalactan (AG) is a natural branched polysaccharide with a molecular mass near $16 \mathrm{kDa}$ extracted from Western or Siberian Larch, consisting of arabinose and galactose monosaccharides. AG is highly water-soluble, non-toxic, biodegradable and biocompatible, contains different functional groups such as hydroxyl, carboxylic acid, that make it suitable for conjugation and delivery of carotenoids and other drug molecules. To study the reactivity of carotenoid incorporated into AG macromolecule in aqueous solution, we have applied EPR and

Fig. 9 EPR spectra of PBN$\mathrm{OOH}$ spin adduct in the presence of HP- $\beta$-CD complex of 8 -apo- $\beta$-caroten- 8 '-oic acid. Concentration of $\mathrm{PBN}=10 \mathrm{mM}$; $\mathrm{Fe}^{2+}=1 \mathrm{mM} ; \mathrm{H}_{2} \mathrm{O}_{2}=500 \mathrm{mM}$; $\mathrm{HP}-\beta-\mathrm{CD}=4 \mathrm{mM}$ in $\mathrm{H}_{2} \mathrm{O}$ (from Ref. [36])

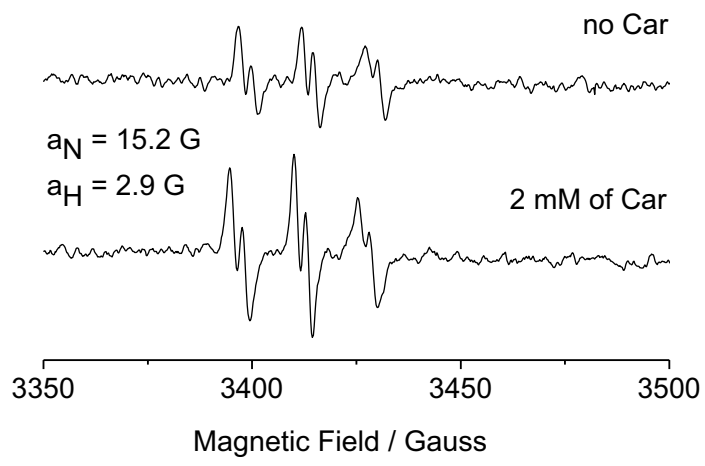


optical spectroscopy techniques. The carotenoid-AG complexes maintain their original color and show insignificant changes in absorption spectra [23, 45, 48]. It was demonstrated also that incorporation of xanthophyll carotenoids into AG macromolecule prevents $\mathrm{H}$-aggregates formation of these carotenoids in the presence of water. The important feature of the carotenoid-AG complexes is enhanced photo stability and oxidation stability compared to pure carotenoids [23, 45, 48]. About one order increase in the photostability of carotenoids lutein, astaxanthin and canthaxanthin has been detected. As an example, Fig. 10 shows the decrease in the photodegradation rate of carotenoid astaxanthin in AG complex.

The carotenoid-AG complexes show also the enhancement of chemical stability. The complete inhibition of oxidation of the carotenoids lutein and zeaxanthin by ferric ions as an electron acceptor and by ozone molecules as a powerful oxidant has been demonstrated in our study [48]. Oxidation of carotenoids by ferric ions results in the formation of the carotenoid radical cations which are unstable in aqueous solutions due to fast deprotonation and formation of neutral radicals [52]. We assume two main factors responsible for chemical and photochemical stabilization of carotenoids in the AG complexes, namely, isolation from water molecules, which serve as a proton acceptor from carotenoid radical cations, and isolation from reactive species (ozone molecules and metal ions).

An interesting application of the carotenoid-AG complexes has been demonstrated in the field of photocatalysis [50]. Photoirradiation of $\mathrm{TiO}_{2}$ nanoparticles in the presence of beta-carotene-AG complex by visible light enhance the yield of the reactive hydroxyl radicals detected by the EPR spin-trapping technique (Fig. 11). The free radicals formed in this system proceed via oxygen reduction on the $\mathrm{TiO}_{2}$ surface. The observed enhancement of the photocatalytic efficiency for carotenoid complexes, as measured by the quantum yield of the desired spinadducts, arises specifically from the decrease in the rate constant for the back electron transfer to the carotenoid radical cation. These results are important for a variety of $\mathrm{TiO}_{2}$ applications, in particular in the design of artificial light-harvesting, photoredox, and catalytic devices.

Fig. 10 Photodegradation of astaxanthin in aerated waterethanol solution under the full light of a xenon lamp. Irradiation time $1 \mathrm{~min}$ (adopted from [23])

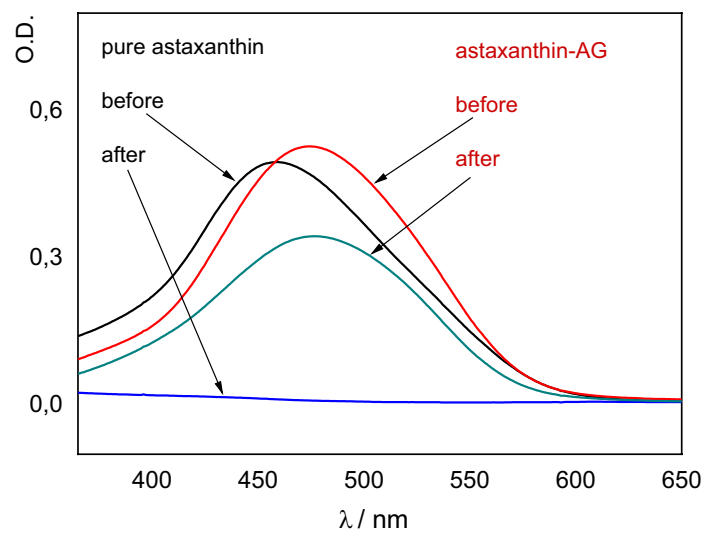


Fig. 11 EPR spectra of PBN$\mathrm{CH}_{3}$ spin adducts formed during irradiation of a water suspension of $\mathrm{TiO}_{2}$ nanoparticles in the absence (upper) and in the presence of $\beta$-carotene-AG complex (lower) with $\lambda>350 \mathrm{~nm}$ in aqueous solution in the presence of $10 \%$ DMSO (adopted from [50])

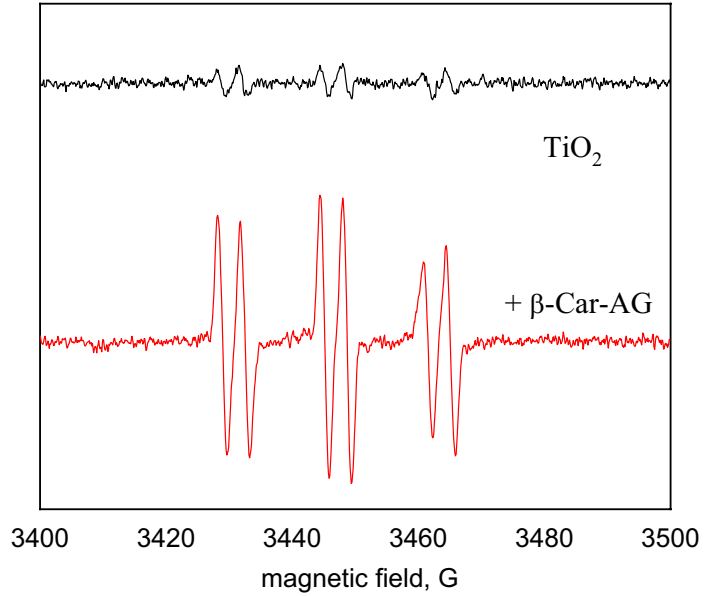

Glycyrrhizic acid (GA) is a saponin extracted from licorice root. GA is an amphiphilic molecule that contains the hydrophilic part-glucuronic acid residues, and the hydrophobic part-glycyrrhetinic acid residue. Application of GA in medicine has a very long history, licorice was used in traditional medicine since ancient times in China, Egypt and Japan [44]. Nowadays, the biological and therapeutic activity of GA is still intensively investigated, in particular its anti-coronavirus activity [53]. About 15 years ago a novel unusual property of the GA has been discovered, namely its ability to form stable self-associates (dimers and micelles) [44, 54-56] which are able to form water-soluble inclusion complexes with other lipophilic molecules including carotenoids [46-49]. In addition, the membrane modifying ability of GA has been described [57-61] and considered as the key factor of its therapeutic activity, including anticancer and antivirus activities.

Supramolecular complexes of beta-carotene, canthaxanthin, lutein, zeaxanthin, astaxanthin and other carotenoids with GA were studied by various physicochemical methods including NMR and EPR spectroscopy, electrochemistry and UV-Vis optical spectroscopy [23, 46-49]. Similar to carotenoid-AG complexes, the encapsulation of carotenoids lutein and zeaxanthin into GA micelles protects these carotenoids from oxidation by reactive oxygen species $\left(\mathrm{O}_{3}\right.$ and $\mathrm{OH}$ radical $)$ and metal ions [23, 48], and decrease the oxidation rate of these carotenoids by 10-20 times.

In contrast to previously studied water-soluble oligosaccharides and polysaccharides, GA is able to form supramolecular complexes with carotenoids not only in aqueous solutions where GA complexes increased the carotenoid solubility more than 1000-fold [48], but also in non-aqueous organic solvents (alcohols, DMSO, acetonitrile) [46, 47]. This fact is important for discussion the possibility of GAassisted transport of carotenoid molecules through lipophilic cell membranes and their membrane protection properties. It was found, that in non-aqueous media, GA is able to form non-covalent complexes not only with neutral carotenoid molecules but also with their radical cations and charge transfer complexes with electron donors [46]. The 50-fold increase of the lifetime of $\beta$-carotene radical cation in the presence of GA has been demonstrated. High stability of the carotenoid radical 
cations imbedded into GA host opens possibilities for the application of these complexes for the design of artificial light-harvesting, photoredox and catalytic systems.

Taking into account the important role of carotenoids as antioxidants in living systems, the antioxidant activity of carotenoid-GA complexes was studied by the EPR spin-trapping technique. Comparison of the scavenging rates of $\mathrm{OOH}$ radicals by a set of carotenoids and their GA complexes in DMSO solution shows a strong dependence of the rate constants on the carotenoids structure and their oxidation potentials [47]. Relative scavenging rate constant of $\mathrm{OOH}$ radical by carotenoids beta-carotene and zeaxanthin does not change in the presence of GA but increased by factor of 15 for carotenoid canthaxanthin and by factor of 5 for carotenoid 7-apo7,7 -dicyano- $\beta$-carotene in the presence of GA [47]. Since it was found that scavenging ability of carotenoids towards $\mathrm{OOH}$ radicals is potential dependent $[22,25]$ we have tested the effect of GA on the oxidation potential of carotenoids zeaxanthin and canthaxanthin. In both cases, an increase in $E_{1 / 2}$ by $0.03-0.05 \mathrm{~V}$ was observed [47] (Fig. 12).

Recent measurements of the oxidation potential of the carotenoid bixin extracted from Annatto seeds exhibits the highest oxidation potential of all carotenoids, $0.94 \mathrm{~V}$ [63]. According to the plot in Fig. 12, this large oxidation potential predicts bixin to be the best radical scavenger to date. This is consistent with the use of bixin in food, cosmetics and pharmaceuticals to increase their shelf life since it was approved by FDA in 1938.

We assume that interaction between carotenoids and $\mathrm{OOH}$ radicals occurs via hydrogen abstraction from the most acidic 4-H proton of carotenoids [32]. GA forms a donut-like dimer in which the polyene chain of carotenoids lies protected within the donut hole, permitting the hydrophilic ends to be exposed to the surroundings. In contrast, in the case of the carotenoid-CD complexes, the terminal group of the carotenoid is completely protected which results in the inhibition of any antioxidant activity [36].

Fig. 12 The dependence of the carotenoids scavenging rate toward $\mathrm{OOH}$ radicals on the oxidation potential of carotenoids. Arrows denote the shifts in oxidation potentials due to complexation with glycyrrhizic acid [62]

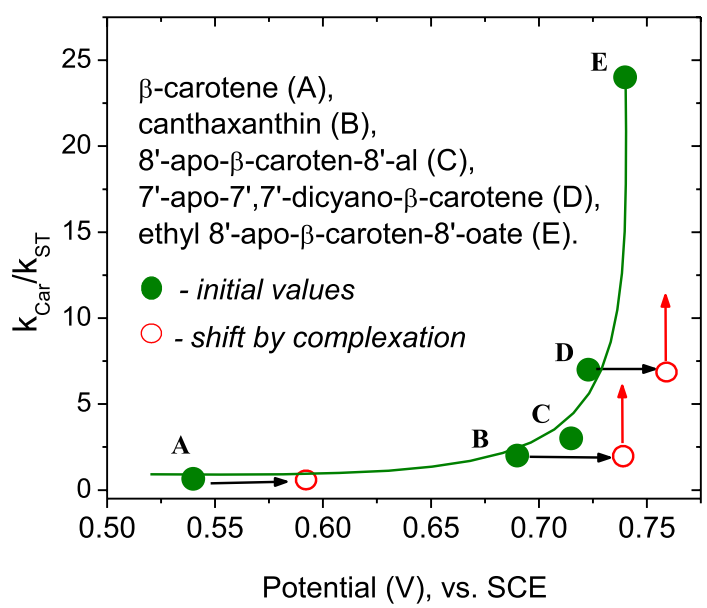

Springer 


\section{Conclusion}

In this mini-review, we discussed the results of numerous physicochemical studies of carotenoid radicals, namely the radical cations formed by electron transfer and neutral radicals formed by proton loss from the radical cations. These radicals were detected and characterized in solution, powders and in vivo in LHCII. Various EPR techniques such as CW ENDOR and pulsed methods like Davies and Mims ENDOR in combination with DFT calculations of the hyperfine couplings were used to distinguish and identify these radical species in catalysts containing silanol groups. As a consequence of deprotonation of the radical cation, the unpaired electron spin distribution changes so that larger isotropic $\beta$-methyl proton couplings occur for the neutral radicals Car $(13-16 \mathrm{MHz})$ than for the radical cation $\mathrm{Car}^{+}(7-10 \mathrm{MHz})$, providing a means to differentiate between these carotenoid radicals. The hypothesis of the photoprotective role of carotenoid neutral radicals formed by proton loss from the radical cation is described herein. The radical cation is already known to participate in the quenching of excited states of chlorophyll so its deprotonation would be favored near water molecules, or near aqueous media like stroma and lumen outside the thylakoid membrane.

EPR technique in combination with other physicochemical methods has also aided in elucidating properties of carotenoids incorporated in different hosts. We have demonstrated that incorporation of carotenoids into water-soluble carriers such as oligosaccharide cyclodextrin, polysaccharide arabinogalactan and saponin glycyrrhizin improved carotenoids' solubility and some of the carriers improved carotenoids' stability, including photostability and oxidation stability. Importantly, the encapsulation of carotenoids into the host cavity affects the fundamental properties of carotenoids such as redox potentials, antioxidant activity, as well as the yield and lifetime of paramagnetic forms of carotenoids.

Acknowledgements A. L. F. would like to acknowledge support from the Faculty Research Seed Grants (FRSG) Program at Valdosta State University. The reported research was funded by the Russian Ministry of Science and Education (State assignments no. 0304-2017-0009 and 0301-2019-0005 (N. E. P), by the National Science Foundation with EPR instrument grants CHE-0342921 and CHE-0079498 (L. D. K.) and by the Chemical Sciences, Geosciences and Biosciences Division, Office of Basic Sciences, U.S. Department of Energy, grant DEFG02-86ER-13465 for lab supplies (L. D. K.).

\section{References}

1. S.T. Mayne, B. Cartmel, M. Baum, G. Shor-Posner, B.G. Fallon, K. Briskin, J. Bean, T. Zheng, D. Cooper, C. Friedman, W.J. Goodwin Jr., Cancer Res. 61, 1457-1463 (2001)

2. R. Goralczyk, Nutr. Cancer 61, 767-774 (2009)

3. A. Green, G. Williams, R. Neale, V. Hart, D. Leslie, P. Parsons, G.C. Marks, P. Gaffney, D. Battistutta, C. Frost, C. Lang, Lancet 354, 723-729 (1999)

4. E. Leoncini, V. Edefonti, M. Hashibe, M. Parpinel, G. Cadoni, M. Ferraroni, D. Serraino, A.F. Olshan, J.P. Zevallos, D.M. Winn, K. Moysich, Z.F. Zhang, H. Morgenstern, F. Levi, K. Kelsey, M. McClean, C. Bosetti, S. Schantz, G.P. Yu, P. Boffetta, Y.C. Lee, S.C. Chuang, A. Decarli, C. La Vecchia, S. Boccia, Eur. J. Epidemiol. 31, 369-383 (2016) 
5. A.R. Vieira, L. Abar, S. Vingeliene, D.S.M. Chan, D. Aune, D. Navarro-Rosenblatt, C. Stevens, D. Greenwood, T. Norat, Ann. Oncol. 27, 81-96 (2016)

6. L. Feng, K. Nie, H. Jiang, W. Fan, PLoS ONE 14, e0227048 (2019)

7. T.H.P. Brotosudarmo, L. Limantara, E. Setiyono, H. Heriyanto, Int. J. Food Sci. 10, 1-16 (2020)

8. T. Zhao, X. Yan, L. Sun, T. Yang, X. Hu, Z. He, F. Liu, X. Liu, Trends Food Sci. Technol. 91, 354-361 (2019)

9. Y. Gao, A.L. Focsan, L.D. Kispert, Antioxidants 9, 625 (2020)

10. P. Hapiot, L.D. Kispert, V.V. Konovalov, J.-M. Saveant, J. Am. Chem. Soc. 123, 6669-6667 (2001)

11. A.L. Focsan, S. Pan, L.D. Kispert, J. Phys. Chem. B 118, 2331-2339 (2014)

12. Y. Gao, A.L. Focsan, L.D. Kispert, Chem. Phys. Lett. 761, 138098 (2020)

13. J.A. Jeevarajan, L.D. Kispert, J. Electroanal. Chem. 411, 57-66 (1996)

14. A.L. Focsan, P. Molnár, J. Deli, L. Kispert, J. Phys. Chem. B 113, 6087-6096 (2009)

15. A.L. Focsan, M.K. Bowman, T.A. Konovalova, P. Molnár, J. Deli, D.A. Dixon, L.D. Kispert, J. Phys. Chem. B 112, 1806-1819 (2008)

16. A.L. Focsan, L.D. Kispert, J. Photochem. Photobiol. B Biol. 166, 148-157 (2017)

17. A.L. Focsan, N.E. Polyakov, L.D. Kispert, Antioxidants 6, 80 (2017)

18. P.L. Hasjim, F. Lendzian, N. Ponomarenko, J.R. Norris, J. Phys. Chem. Lett. 1, 1687-1689 (2010)

19. A.L. Focsan, A. Magyar, L.D. Kispert, Arch. Biochem. Biophys. 572, 167-174 (2015)

20. E. Hideg, C. Barta, T. Kalai, I. Vass, K. Hideg, K. Asada, Plant Cell Physiol. 43, 1154-1164 (2002)

21. A. Magyar, M.K. Bowman, P. Molnár, L. Kispert, J. Phys. Chem. B 117, 2239-2246 (2013)

22. N.E. Polyakov, A.I. Kruppa, T.V. Leshina, T.A. Konovalova, L.D. Kispert, Free Radic. Biol. Med. 31, 43-52 (2001)

23. N.E. Polyakov, A. Magyar, L.D. Kispert, J. Phys. Chem. B. 117, 10173-10182 (2013)

24. D. Niedzwiedzki, J.F. Rusling, H.A. Frank, Chem. Phys. Lett. 415, 308-312 (2005)

25. N.E. Polyakov, T.V. Leshina, T.A. Konovalova, L.D. Kispert, Free Radic. Biol. Med. 31, 398-404 (2001)

26. T.A. Konovalova, L.D. Kispert, J. Chem. Soc. Faraday Trans. 94, 1465-1468 (1998)

27. Y. Gao, A.L. Focsan, L.D. Kispert, D.A. Dixon, J. Phys. Chem. B 110, 24750-24756 (2006)

28. A.S. Jeevarajan, L.D. Kispert, L. Piekara-Sady, Chem. Phys. Lett. 209, 269-274 (1993)

29. T.A. Konovalova, Y. Gao, R. Schad, L.D. Kispert, C.A. Saylor, L.-C. Brunel, J. Phys. Chem. B 105, 7459-7464 (2001)

30. T.A. Konovalova, Y. Gao, L.D. Kispert, J. van Tol, L.-C. Brunel, J. Phys. Chem. B 107(4), 10061011 (2003)

31. A.L. Focsan, M.K. Bowman, P. Molnar, J. Deli, L.D. Kispert, J. Phys. Chem. B 115, 9495-9506 (2011)

32. A.L. Focsan, M.K. Bowman, J. Shamshina, M.D. Krzyaniak, A. Magyar, N.E. Polyakov, L.D. Kispert, J. Phys. Chem. B 116, 13200-13210 (2012)

33. K. Barbusiński, Ecol. Chem. Eng. S 16, 347-358 (2009)

34. D. Liu, Y. Gao, L.D. Kispert, J. Electroanal. Chem. 488, 140-150 (2000)

35. D. Liu, L.D. Kispert, Recent Res. Dev. Electrochem. 2, 139-157 (1999)

36. N.E. Polyakov, T.V. Leshina, T.A. Konovalova, E.O. Hand, L.D. Kispert, Free Radic. Biol. Med. 36, 872-880 (2004)

37. V. Timoshnikov, T. Kobzeva, N. Polyakov, G.J. Kontoghiorghes, Int. J. Mol. Sci. 21, 3967 (2020)

38. V.A. Timoshnikov, T.V. Kobzeva, OYu. Selyutina, N.E. Polyakov, G.J. Kontoghiorghes, J. Biol. Inorg. Chem. 24, 331-341 (2019)

39. N.E. Polyakov, L.D. Kispert, Carbohydr. Polym. 128, 207-219 (2015)

40. S. Akhavan, E. Assadpour, I. Katouzian, S.H. Jafari, Trends Food Sci. Technol. 74, 132-146 (2018)

41. I. Katouzian, F. Esfanjani, S.M. Jafari, S. Akhavan, Trends Food Sci. Technol. 68, 14-25 (2017)

42. A.L. Focsan, N.E. Polyakov, L.D. Kispert, Molecules 24, 3947 (2019)

43. C. Soukoulis, T.A. Bohn, Crit. Rev. Food Sci. Nutr. 58, 1-36 (2018)

44. OYu. Selyutina, N.E. Polyakov, Int. J. Pharm. 559, 271-279 (2019)

45. N.E. Polyakov, T.V. Leshina, E.S. Meteleva, A.V. Dushkin, T.A. Konovalova, L.D. Kispert, J. Phys. Chem. B 113, 275-282 (2009)

46. N.E. Polyakov, T.V. Leshina, N.F. Salakhutdinov, L.D. Kispert, J. Phys. Chem. B 110, 6991-6998 (2006)

47. N.E. Polyakov, T.V. Leshina, N.F. Salakhutdinov, T.A. Konovalova, L.D. Kispert, Free Radic. Biol. Med. 40, 1804-1809 (2006) 
48. I.E. Apanasenko, O.Y. Selyutina, N.E. Polyakov, L.P. Suntsova, E.S. Meteleva, A.V. Dushkin, P. Vachali, P.S. Bernstein, Arch. Biochem. Biophys. 572, 58-65 (2015)

49. B. Li, P.P. Vachali, Z. Shen, A. Gorusupudi, K. Nelson, B.M. Besch, A. Bartschi, S. Longo, T. Mattinson, S. Shihab, N.E. Polyakov, L.P. Suntsova, A.V. Dushkin, P.S. Bernstein, Exp. Eye Res. 159, 123-131 (2017)

50. N.E. Polyakov, T.V. Leshina, E.S. Meteleva, A.V. Dushkin, T.A. Konovalova, L.D. Kispert, J. Phys. Chem. B 114, 14200-14204 (2010)

51. T. Higashi, Chem. Pharm. Bull. 67, 289-298 (2019)

52. Y. Gao, S. Webb, L.D. Kispert, J. Phys. Chem. B 107, 13237-13240 (2003)

53. M.N.M.B. Younes, A.D. Alshawabkeh, Z.A. Al-Masalha, T.T. Malhis, Z.G.A. Alameri, T.M. Al Tarabsheh, EAS J. Pharm. Pharmacol. 2, 31-35 (2020)

54. S.S. Petrova, A.A. Schlotgauer, A.I. Kruppa, T.V. Leshina, Z. Phys. Chem. 231, 1-17 (2016)

55. S.N. Borisenko, A.V. Lekar, A.A. Milov, E.V. Vetrova, N.I. Borisenko, Chem. Plant Mater. 2, 85-92 (2013)

56. A.V. Dushkin, E.S. Meteleva, T.G. Tolstikova, M.V. Khvostov, M.P. Dolgikh, Chem. Sustain. Dev. 18, 437-444 (2010)

57. O.Y. Selyutina, I.E. Apanasenko, A.G. Shilov, S.S. Khalikov, N.E. Polyakov, Russ. Chem. Bull. 66, 129-135 (2017)

58. O.Y. Selyutina, I.E. Apanasenko, A.V. Kim, E.A. Shelepova, S.S. Khalikov, N.E. Polyakov, Colloids Surf. B Biointerfaces 147, 459-466 (2016)

59. O.Y. Selyutina, N.E. Polyakov, D.V. Korneev, B.N. Zaitsev, Drug Deliv. 23, 858-865 (2016)

60. O.Y. Selyutina, I.E. Apanasenko, N.E. Polyakov, Russ. Chem. Bull. 64, 1555-1559 (2015)

61. O.Y. Selyutina, N.E. Polyakov, D.V. Korneev, B.N. Zaitsev, Russ. Chem. Bull. 63, 1201-1204 (2014)

62. N.E. Polyakov, L.D. Kispert, in Beta Carotene: Dietary Sources, Cancer and Cognition, 1st edn., ed. by L. Haugen, T. Bjornson (Nova Science Publishers, Singapore, 2019), pp. 191-230

63. S. Tay-Agbozo, S. Street, L.D. Kispert, J. Photochem. Photobiol. B Biol. 186, 1-8 (2018)

Publisher's Note Springer Nature remains neutral with regard to jurisdictional claims in published maps and institutional affiliations.

\section{Authors and Affiliations}

\section{A. Ligia Focsan ${ }^{1}$ (D) - Nikolay E. Polyakov ${ }^{2,3} \cdot$ Lowell D. Kispert $^{4}$}

Nikolay E. Polyakov

polyakov@kinetics.nsc.ru

Lowell D. Kispert

lkispert@ua.edu

1 Department of Chemistry, Valdosta State University, Valdosta, GA 31698, USA

2 Institute of Chemical Kinetics and Combustion, Novosibirsk 630090, Russia

3 Institute of Solid State Chemistry and Mechanochemistry, Novosibirsk 630128, Russia

4 Department of Chemistry, The University of Alabama, Box 870336, Tuscaloosa, AL 35487, USA 